\title{
Factors associated with ocular surface epithelial damage in patients with primary Sjögren's syndrome
}

\author{
Ji Eun Kim, Na Rae Kim', Hee Seung Chin ${ }^{1}$, Kyoung Yul Seo ${ }^{2}$, Tae-im Kim² and Ji Won Jung ${ }^{1 *}$
}

\begin{abstract}
Background: The aim of this study was to evaluate the effects of systemic parameters, laboratory findings, oral parameters, and other ocular surface parameters on ocular surface epithelial damage in patients with primary Sjögren's syndrome (pSS).

Methods: A total of 82 dry eye disease (DED) patients with pSS were enrolled in this study. Ocular surface epithelial damage was measured by ocular staining score (OSS). Systemic parameters, laboratory findings including serologic markers, oral parameters, and other ocular surface parameters were collected. Other ocular surface parameter assessments such as the Schirmer's test, fluorescein tear breakup time, meibomian gland examinations, noninvasive keratographic tear film break-up time measurements using the Keratograph ${ }^{\circledast} 5 \mathrm{M}$ were performed, and the Ocular Surface Disease Index was determined.

Results: In a multivariate analysis, decreased age and increased duration of pSS were significantly related to increased logarithm-transformed OSS ( $\beta=-0.011, P=0.043$ and $\beta=0.003, P=0.008$ ). Among the ocular surface parameters, decreased fluorescein tear breakup time and increased MGD grade were significantly associated with increased logarithm-transformed OSS $(\beta=-0.183, P<0.001$ and $\beta=0.192, P=0.049)$.
\end{abstract}

Conclusions: Ocular surface epithelial damage in patients with pSS was associated with young age, long duration of disease, unstable tear film, and decreased meibomian gland function.

Keywords: Dry eye disease, Primary Sjögren's syndrome, Ocular surface epithelial damage, Ocular staining score, Fluorescein tear break up time, Meibomian gland dysfunction

\section{Background}

Dry eye disease (DED) is a multifactorial ocular surface disease characterized by a loss of tear film homeostasis $[1,2]$. In the 2017 Tear Film and Ocular Surface society (TFOS) Dry Eye Workshop (DEWS) II report, DED was classified into aqueous deficient dry eye (ADDE) and evaporative dry eye. ADDE is categorized into two groups, Sjögren's syndrome (SS)-related dry eye and non-SS DED [2]. In patients with significant ADDE,

\footnotetext{
* Correspondence: panch325@gmail.com

${ }^{1}$ Department of Ophthalmology and Inha Vision Science Laboratory, Inha University School of Medicine, 27, Inhang-Ro, Jung-gu, 22332 Incheon, South Korea

Full list of author information is available at the end of the article
}

$11.6 \%$ had SS: $6.4 \%$ had primary SS (pSS), and $5.2 \%$ had secondary SS [3].

$\mathrm{SS}$ is a systemic autoimmune disease, in which T cells and autoantibody producing B cells infiltrate exocrine glands, such as salivary gland and lacrimal gland [4-6]. Th1- and Th17-associated cytokines, IFN- $\gamma$, and IL-17, are all associated with increased inflammation and glandular dysfunction, and IL-1 suppress lacrimal acinar secretion [7-9]. This inflammation also involves the extragland, which causes symptoms of pain, myalgia, and inflammation of the joint, vascular system, skin, lungs, and kidneys [10]. When SS occurs by itself, it is referred to as pSS, and when accompanied by another autoimmune disease, it is referred to as secondary

(c) The Author(s). 2021 Open Access This article is licensed under a Creative Commons Attribution 4.0 International License, which permits use, sharing, adaptation, distribution and reproduction in any medium or format, as long as you give appropriate credit to the original author(s) and the source, provide a link to the Creative Commons licence, and indicate if changes were made. The images or other third party material in this article are included in the article's Creative Commons licence, unless indicated otherwise in a credit line to the material. If material is not included in the article's Creative Commons licence and your intended use is not permitted by statutory regulation or exceeds the permitted use, you will need to obtain permission directly from the copyright holder. To view a copy of this licence, visit http://creativecommons.org/licenses/by/4.0/ The Creative Commons Public Domain Dedication waiver (http://creativecommons.org/publicdomain/zero/1.0/) applies to the data made available in this article, unless otherwise stated in a credit line to the data. 
SS [11]. The diagnosis of pSS is made in combination with at least 1 symptom of ocular or oral dryness or systemic manifestations as well as signs such as ADDE findings; decreased tear secretion and significant ocular staining score (OSS), the presence of autoantibodies, evidence of reduced salivary gland secretion, and positive findings of minor salivary gland biopsy [12].

In DED, excessive dryness causes tear film hyperosmolarity, which stimulates the production of cytokines from the ocular surface epithelium [13]. This process activates an inflammatory cascade at the ocular surface, which leads to corneal barrier disruption and conjunctival goblet cell dysfunction [13]. The consequent ocular surface epithelial damage can be seen in severe DED and as an indicator of ocular surface epithelial damage, and the OSS has been demonstrated to be an informative marker of disease severity of DED [14]. Also, the OSS showed positive correlations with the expression of inflammatory cytokines in SS related DED, which is an important marker of ocular surface inflammation [13]. Previous few studies have also reported an association between the OSS and not only ocular surface inflammation, but also systemic parameters such as positive serologic findings $[15,16]$.

The factors that affect ocular surface epithelial damage in the patients with pSS (Fig. 1a, b) have not been well studied yet. Furthermore, this type of damage is important in determining optimal treatment strategies for DED patients with pSS. Therefore, the purpose of our study was to evaluate the effects of systemic parameters, laboratory findings, oral parameters, and other ocular surface parameters on ocular surface epithelial damage in patients with pSS by a multivariate analysis.

\section{Methods}

\section{Subjects}

The study was approved by the Institutional Review Board (IRB) of Inha University Hospital and it was complied with the tenets of the Declaration of Helsinki. No written informed consent was required as data was retrospectively collected from chart review, as approved by the reference IRB.

This retrospective chart review of patients with SS among outpatients who visited for management of dry eye symptoms from March 2015 to July 2019 in the Department of Ophthalmology at Inha University Hospital. One hundred thirty-five SS patients with recorded ocular surface parameters (including the OSS) and checkable medical chart were analyzed, and 73 patients who were excluded based on the pSS criteria. A total of 82 patients with DED who were diagnosed with pSS were enrolled in this study. The American College of Rheumatology/ European League against Rheumatism classification criteria for rheumatoid arthritis (ACR/EULAR) was used to diagnose pSS [17], which was defined as a score $\geq 4$ with at least 1 symptom of ocular or oral dryness or the presence of systemic manifestations of SS. We excluded patients younger than 20 years of age as well as those with histories of ocular surgery within 6 months, ocular injury, or other ocular diseases.

\section{Outcome measures}

The clinical variables, presence of systemic disease, and previous medical history were investigated. The age was defined as age at the time of examination of OSS, and the duration of pSS was defined as the period from the diagnosis of pSS to the examination of OSS. We assessed the current medication use at the time of examination of OSS, at least 3 months of use were included. Oral medications such as pilocarpine, hydroxychloroquine, methotrexate, cyclosporine, and steroid for SS, and topical medications such as cyclosporine and steroid for DED were included. The duration of the topical cyclosporine use was 13.2 months (range, 6-25 months) and topical steroid use was 11.3 months (range, 3-18 months).

Laboratory parameters included white blood cell, erythrocyte sedimentation rate, C-reactive protein, complement $\mathrm{C} 3$, complement $\mathrm{C} 4$, and immunoglobulin $\mathrm{G}$.
(A)

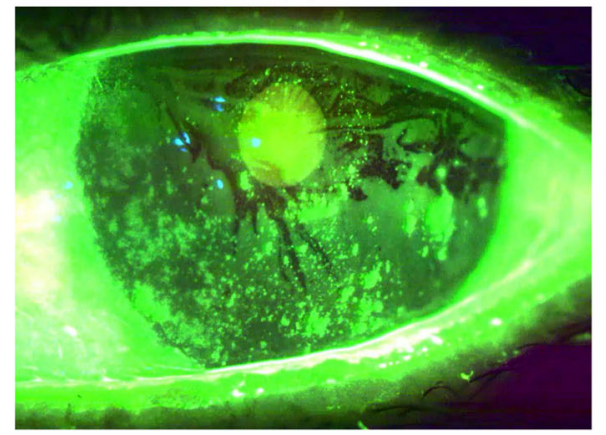

(B)

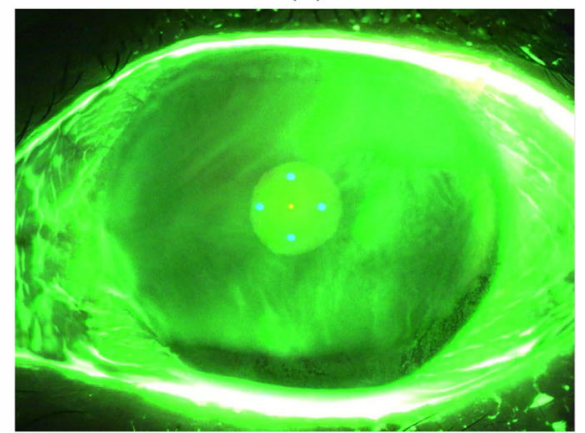

Fig. 1 The K5M images of ocular surface staining in two dry eye patients with primary Sjögren's syndrome (pSS). a Severe ocular surface staining in a 60-year-old woman with pSS. b Mild ocular surface staining in a 59-year-old woman with pSS 
The positivity of antibodies associated with connective tissue diseases, such as anti-Sjögren's-syndrome-related antigen $\mathrm{A}(\mathrm{SSA}) / \mathrm{Ro}$, anti- Sjögren's-syndrome-related antigen $\mathrm{B}(\mathrm{SSB}) / \mathrm{La}$, antinuclear antibody (ANA), and rheumatoid factor (RF), were assessed. The positive ranges of antibodies are as follows: anti-SSA/Ro antibody $>10 \mathrm{U} / \mathrm{mL}$, anti-SSB/La antibody $>10 \mathrm{U} / \mathrm{mL}$, ANA $\geq 1: 80$, and $R F>14.0 \mathrm{IU} / \mathrm{mL}$. All laboratory parameters were measured at the Inha University Hospital laboratory medicine department, and the laboratory findings closest to the time of examination of OSS at Inha University hospital ophthalmology outpatient clinic were used. The average time interval between the laboratory findings and examination of OSS was $6.1 \pm 6.6$ months.

Ocular surface parameters were performed as follows and data were obtained from the right eye unless the right eye was excluded from the study, in which case data were collected from the left eye. (1) OSS according to the National Eye Institute/Industry Workshop scale [17-20], which was graded from 0 to 3 for each of the five areas on cornea and each of the six areas on conjunctiva. A total score from 0 to 33 based on the pattern of fluorescein staining observed under the slit lamp. (2) Subjective symptoms were graded on a numerical scale from 0 to 4 , according to the validated 12-item ocular surface disease index (OSDI) questionnaire. The total OSDI, ranging from 0 to 100 , was calculated using the following formula: OSDI $=$ (sum of scores for all questions answered $\times 100$ ) / (total number of answered questions $\times 4) \quad[18,20-22]$. (3) Schirmer's test I was performed without topical anesthesia by placing a Schirmer strip in the mid-lateral portion of the lower fornix. The amount of wetting was recorded after $5 \mathrm{~min}$, and the patients were asked to keep their eyes lightly closed during the test. (4) Fluorescein break-up time (FBUT) was measured through application of a single fluorescein strip (Haag-Streit, Koeniz, Switzerland) to the inferior palpebral conjunctiva after instilling a drop of normal saline. The mean time for three results was recorded. (5) The lid margins and meibomian glands were checked for lid margin abnormalities, gland expression, and meibum quality, as previously described [20, 21, 23-27]. Lid margin abnormalities were scored as 0 (absent) or 1 (present) for the following parameters: vascular engorgement, plugged meibomian gland orifices, anterior or posterior displacement of the mucocutaneous junction, and irregularity of the lid margin [21, 23-25, 27]. The presence of an inflamed lid margin was checked. The degree of meibomian gland expressibility using firm digital pressure applied on five glands of the central third of the lower lid was graded as follows: grade 0, all five glands expressible; grade 1, three to four glands expressible; grade 2, one to two glands expressible; and grade 3, no glands expressible [24, 26, 27]. The meibum quality over eight lower lid glands was graded as follows: grade 0 , clear; grade 1, cloudy; grade 2, cloudy with granular debris; and grade 3, thick (texture similar to toothpaste). Each of the eight glands of the lower eyelid was graded on a scale from 0 to 3 . The scores of the eight glands were summed to obtain a total score (range, 0-24) [21, $24,27]$. The meibomian gland dysfunction (MGD) stage was determined based on the three lid parameters: MGD stage 1 , minimally altered expressibility (grade 1 ) and secretion quality $(2 \leq$ grade $<4)$; MGD stage 2 , scattered lid margin features, mildly altered expressibility (grade 1 ) and secretion quality $(4 \leq$ grade $<8)$; MGD stage 3 , lid margin features of plugging, vascularity, moderately altered expressibility (grade 2$)$ and secretion quality $(8 \leq$ grade $<13$ ); MGD stage 4 , lid margin features of dropout, displacement, severely altered expressibility (grade 3 ), and secretion quality (grade $\geq 13$ ) [23, 27]. (6) Tear film assessment with the "TF-Scan, Non-Invasive Keratograph ${ }^{\circ}$ Break-Up Time (NIKBUT) mode" was conducted when the subjects were instructed to blink three or four times and then keep their eyes open for as long as possible. Irregularities on the image indicated instability or breakup of the tear film and, at the same time, a video was recorded. The device provided a representation of tear film break up over time, including a tear film map showing the location and size of the tear film-break regions, as well as the first break-up time (NIKBUTfirst) and the average break-up time (NIKBUT-average; the mean of all tear film break-ups occurring over the entire cornea), as previously described [20, 28, 29].

Oral parameters included subjective oral symptom score, non-stimulated whole salivary flow (NSWSF), and positivity of a minor salivary gland biopsy. The subjective oral symptom score was based on the 2002 American-European Consensus Group (AECG) criteria, and three questions were summed to obtain a total score (range, 0-3): (1) symptoms of dry mouth for at least 3 months, (2) recurrent or persistently swollen salivary glands, and (3) need for liquids to swallow dry foods [17]. NSWSF reflects the basal flow from all glands. Patients must swallow any residual saliva before starting the procedure and allow all saliva to accumulate in the mouth and spit it every minute. Saliva was collected for 15 minutes and the measured volume was expressed in $\mathrm{mL} /$ $\mathrm{min}$. For the SS criteria, a value $\leq 0.1 \mathrm{~mL} / \mathrm{min}$ was considered abnormal $[17,30]$. Minor salivary gland biopsy positivity was determined based on the AECG criteria, which was defined as a focus score of 1 or more. Focus was defined as a dense aggregate of 50 or more lymphocytes in a 4- $\mathrm{mm}^{2}$ area of the glandular tissue [17].

\section{Statistical analyses}

Statistical analyses were performed using SPSS for Windows (version 20.0; SPSS Inc., Chicago, IL, USA). The 
OSS was logarithm-transformed to be approximately normally distributed. Univariate and multivariate linear regression analyses were computed to evaluate the impact of systemic and laboratory parameters, oral parameters, and other ocular surface parameters on the logarithm-transformed OSS. For the multivariate linear regression analysis, age, sex, and other variables with $P$ value less than 0.1 in the univariate model were included. In all tests, a $P$ value less than 0.05 was considered significant.

\section{Results}

A total of 82 DED patients with pSS were included in this study. Table 1 shows the clinical variables, presence of systemic disease, and previous medical history of 82 patients. The mean age was 53.8 years old (range, 2480 years), and 78 patients $(95.1 \%)$ were women. The mean duration of pSS was 39.4 months (range, 1185 months). The laboratory findings and oral parameters of all 82 patients are summarized in Table 2: $77.2 \%$ of the patients were positive for anti-SSA/Ro, $38.6 \%$ were positive for anti-SSB/La, and the proportion of both positive were $41.5 \%$. 63.4\% were positive for ANA, and $43.8 \%$ were positive for RF. The ocular surface

Table 1 The systemic parameters and univariate analysis of these parameters on logarithm transformed ocular staining score

\begin{tabular}{|c|c|c|c|}
\hline \multirow[t]{2}{*}{ Variables } & \multirow{2}{*}{$\begin{array}{l}\text { DED related to } \\
\text { pSS }(n=82)\end{array}$} & \multicolumn{2}{|c|}{ Univariate analysis } \\
\hline & & Beta (SE) & $P$-value \\
\hline Age (y) & $53.8 \pm 11.2(24-80)$ & $0.009(0.006)$ & 0.136 \\
\hline \multicolumn{4}{|l|}{ Sex, n (\%) } \\
\hline Male & $4(4.9 \%)$ & 1 (Ref) & \\
\hline Female & $78(95.1 \%)$ & $0.005(0.309)$ & 0.988 \\
\hline Duration of disease (m) & $39.4 \pm 45.4(1-185)$ & $0.003(0.001)$ & 0.029 \\
\hline \multicolumn{4}{|l|}{ Systemic disease, n (\%) } \\
\hline No history & $61(74.4 \%)$ & 1 (Ref) & \\
\hline Hypertension & $16(19.5 \%)$ & $0.144(0.167)$ & 0.389 \\
\hline Diabetes mellitus & $3(3.7 \%)$ & $0.250(0.353)$ & 0.482 \\
\hline \multicolumn{4}{|l|}{ Ocular surgery history, n (\%) } \\
\hline No history & $69(84.1 \%)$ & 1 (Ref) & \\
\hline Refractive surgery & $6(7.3 \%)$ & $-0.356(0.252)$ & 0.162 \\
\hline \multicolumn{4}{|l|}{ Pharmacological history, n (\%) } \\
\hline Oral Pilocarpine & $16(19.5 \%)$ & $-0.024(0.134)$ & 0.857 \\
\hline Oral Hydroxychloroquine & $3(3.7 \%)$ & $-0.134(0.147)$ & 0.365 \\
\hline Oral Methotrexate & $15(18.3 \%)$ & $-0.029(0.172)$ & 0.864 \\
\hline Oral Cyclosporine & $3(3.7 \%)$ & $0.413(0.351)$ & 0.243 \\
\hline Oral Steroid & $14(17.1 \%)$ & $0.204(0.175)$ & 0.246 \\
\hline Topical Cyclosporine & $3(3.7 \%)$ & $0.551(0.185)$ & 0.004 \\
\hline Topical Steroid & 11 (13.4\%) & $0.218(0.175)$ & 0.217 \\
\hline
\end{tabular}

DED dry eye disease, $p S S$ primary Sjögren's syndrome, SE standard error parameters and keratographic parameters of the $82 \mathrm{pa}$ tients are summarized in Table 3: the mean OSS was $7.65 \pm 5.06$ (range, $0-26$ ).

The results of univariate linear regression analysis are summarized in Tables 1,2 and 3. The duration of pSS, topical cyclosporine history, anti-SSB/La antibody, RF, MGD stage, and subjective oral score were positively associated with logarithm-transformed OSS. FBUT, Schirmer's test-I value, NSWSF were negatively associated with logarithm-transformed OSS (all $P<0.050$ ).

In the multivariate linear regression analysis, age, sex, and statistically significant or borderline significant $(P$ value less than 0.1 ) variables were included. After adjusting for other factors, decreased age and increased duration of pSS were significantly related to increased logarithm-transformed OSS. $(\beta=-0.011, P=0.043$ for age; $\beta=0.003, P=0.008$ for duration of $\mathrm{pSS}$ ). After adjusting for other factors, positive serology or oral parameters was not related to OSS in our study. Among the ocular surface parameters, decreased FBUT and increased MGD grade were significantly associated with increased logarithm-transformed OSS $(\beta=-0.183, P<$ 0.001 for FBUT; $\beta=0.192, P=0.049$ for MGD grade) (Table 4).

\section{Discussion}

The aim of this study was to identify the factors associated with ocular surface epithelial damage in patients with pSS. We evaluated the effects of systemic parameters, laboratory findings, oral parameters, and other ocular surface parameters on ocular surface epithelial damage in patients with pSS. Ocular surface epithelial damage was measured by OSS, which is sum of corneal and conjunctival staining score. The OSS is included in the criteria for pSS with Schirmer's test, and it is an informative marker for the severity of DED [12, 14]. Because ocular surface epithelial damage could affect visual disturbance and indicates the presence of ocular surface inflammation, the OSS parameter is important for determining treatment for DED patients with pSS $[13,16$, 31].

Previous studies have reported that an association between dry eye and systemic parameters, or extraocular variables $[11,16,32-35]$. They used the symptoms of ocular dryness, tear osmolarity, or severe DED severity, as indicators of DED. Similar to our study, few studies used the OSS as the main outcome variable, but only reported correlations with laboratory findings, such as positive serologic findings $[15,16]$. Therefore, we focused on the OSS as the main outcome and analyzed its independent association with systemic parameters, laboratory findings, oral parameters, and other ocular surface parameters after adjusting other variables. 
Table 2 Laboratory findings, oral parameters, and univariate analysis of these parameters on logarithm-transformed ocular staining score

\begin{tabular}{|c|c|c|c|}
\hline \multirow[t]{2}{*}{ Variables } & \multirow[t]{2}{*}{ Mean \pm SD } & \multicolumn{2}{|c|}{ Univariate analysis } \\
\hline & & Beta (SE) & $P$-value \\
\hline \multicolumn{4}{|l|}{ Laboratory findings } \\
\hline White blood cell $(/ \mu \mathrm{L})$ & $5122.56 \pm 1496.99$ & $0.000(0.000)$ & 0.350 \\
\hline Erythrocyte sedimentation rate $(\mathrm{mm} / \mathrm{h})$ & $17.09 \pm 13.46$ & $0.006(0.005)$ & 0.262 \\
\hline C-reactive protein (mg/dL) & $0.14 \pm 0.33$ & $0.138(0.203)$ & 0.498 \\
\hline Complement C3 (mg/dL) & $105.68 \pm 19.71\left(68^{\mathrm{a}}\right)$ & $0.003(0.004)$ & 0.471 \\
\hline Complement C4 (mg/dL) & $21.79 \pm 8.32\left(68^{\mathrm{a}}\right)$ & $0.005(0.009)$ & 0.563 \\
\hline Immunoglobulin G (mg/dL) & $1412.61 \pm 407.95\left(38^{\mathrm{a}}\right)$ & $0.000(0.000)$ & 0.124 \\
\hline \multicolumn{4}{|l|}{ Positive serology \% (n) } \\
\hline Anti-SSA/Ro antibody & $77.2\left(61 / 79^{\mathrm{a}}\right)$ & $0.274(0.157)$ & 0.086 \\
\hline Anti-SSB/La antibody & $38.6\left(27 / 70^{a}\right)$ & $0.504(0.140)$ & 0.001 \\
\hline Antinuclear antibody, $(\geq 1: 80)$ & $63.4\left(52 / 82^{\mathrm{a}}\right)$ & $0.235(0.135)$ & 0.087 \\
\hline Rheumatoid factor & $43.8\left(35 / 80^{\mathrm{a}}\right)$ & $0.315(0.132)$ & 0.020 \\
\hline \multicolumn{4}{|l|}{ Oral parameters } \\
\hline Subjective oral score (0-3) & $1.58 \pm 0.71$ & $0.258(0.093)$ & 0.007 \\
\hline Non-stimulated whole salivary flow (mL/min) & $0.09 \pm 0.09\left(79^{\mathrm{a}}\right)$ & $-0.122(0.047)$ & 0.011 \\
\hline Positive results of salivary gland biopsy, \% (n) & $73.7\left(14 / 19^{\mathrm{a}}\right)$ & $0.119(0.293)$ & 0.688 \\
\hline
\end{tabular}

${ }^{a}$ Represented the actual number of patients who underwent the test

SD standard deviation, SSA Sjögren's-syndrome-related antigen A, SSB Sjögren's-syndrome-related antigen B; SE standard error

Among the systemic parameters, age and duration of pSS were significant factors that influenced OSS in the multivariate analysis of our study. A decrease in age was significantly related to an increase in OSS after adjusting for other factors. Although the previous studies of ocular parameters in patients with pSS have reported a correlation with age, they mostly have found a correlation with onset age of pSS or with ocular symptoms other than OSS [32-36]. In their study, the age was negatively related to ocular dryness, indicating older patients with severe disease tended to be less sensitive [32].
In another study, young-onset SS (age at diagnosis < 35 years) correlation with xerostomia $(P=0.008)$, abnormality of Schirmer's test and/or Rose Bengal staining $(P=0.03)$, positivity of anti-Ro/SS-A antibodies $(P<$ 0.001), low complement $C 3(P=0.018)$ and low complement $C 4$ levels $(P=0.017)$, compare to age at diagnosis > 35 years [33].

An increase of the duration of pSS was significantly related to an increase of OSS in the current study. A previous study with a large cohort study of patients with pSS in Spain also reported a correlation between duration of

Table 3 Ocular surface parameters and univariate analysis of these parameters on logarithm-transformed ocular staining score

\begin{tabular}{|c|c|c|c|}
\hline \multirow[t]{2}{*}{ Variables } & \multirow[t]{2}{*}{ Mean \pm SD } & \multicolumn{2}{|c|}{ Univariate analysis } \\
\hline & & Beta (SE) & $P$-value \\
\hline \multicolumn{4}{|l|}{ Ocular surface parameters } \\
\hline Ocular staining score (0-33), NEl scale & $7.65 \pm 5.06$ & & \\
\hline Subjective score (OSDI) & $41.63 \pm 22.88$ & $0.004(0.003)$ & 0.179 \\
\hline Schirmer's test I value (mm) & $7.01 \pm 6.83$ & $-0.038(0.008)$ & $<0.001$ \\
\hline FBUT (seconds) & $2.30 \pm 1.67$ & $-0.230(0.026)$ & $<0.001$ \\
\hline MGD grade $(0-4)$ & $1.95 \pm 0.74$ & $0.432(0.076)$ & $<0.001$ \\
\hline \multicolumn{4}{|l|}{ Parameters using K5M } \\
\hline NIKBUT-first (seconds) & $4.31 \pm 1.73$ & $-0.069(0.035)$ & 0.054 \\
\hline NIKBUT-average (seconds) & $8.20 \pm 3.12$ & $-0.026(0.020)$ & 0.193 \\
\hline
\end{tabular}


Table 4 Univariate and multivariate analysis of variables on logarithm-transformed ocular staining score

\begin{tabular}{|c|c|c|c|c|}
\hline \multirow[t]{2}{*}{ Variables } & \multicolumn{2}{|c|}{ Univariate analysis } & \multicolumn{2}{|c|}{ Multivariate analysis } \\
\hline & Beta (SE) & $\overline{P \text {-value }}$ & Beta (SE) & $P$-value \\
\hline Age (y) & $0.009(0.006)$ & 0.136 & $-0.011(0.005)$ & 0.043 \\
\hline \multicolumn{5}{|l|}{ Sex, n (\%) } \\
\hline Male & 1 (Ref) & & 1 (Ref) & \\
\hline Female & $0.005(0.309)$ & 0.988 & $-0.097(0.250)$ & 0.699 \\
\hline Duration of disease (m) & $0.003(0.001)$ & 0.029 & $0.003(0.001)$ & 0.008 \\
\hline \multicolumn{5}{|l|}{ Pharmacological history, n (\%) } \\
\hline Topical Cyclosporine & $0.551(0.185)$ & 0.004 & $-0.008(0.161)$ & 0.962 \\
\hline \multicolumn{5}{|l|}{ Positive serology \% (n) } \\
\hline Anti-SSA/Ro antibody & $0.274(0.157)$ & 0.086 & $-0.082(0.144)$ & 0.572 \\
\hline Anti-SSB/La antibody & $0.504(0.140)$ & 0.001 & $0.103(0.142)$ & 0.476 \\
\hline Antinuclear antibody, $(\geq 1: 80)$ & $0.235(0.135)$ & 0.087 & $0.191(0.122)$ & 0.123 \\
\hline Rheumatoid factor & $0.315(0.132)$ & 0.020 & $0.096(0.114)$ & 0.404 \\
\hline \multicolumn{5}{|l|}{ Ocular surface parameters } \\
\hline Schirmer's test I value (mm) & $-0.038(0.008)$ & $<0.001$ & $-0.012(0.007)$ & 0.105 \\
\hline FBUT (seconds) & $-0.230(0.026)$ & $<0.001$ & $-0.183(0.033)$ & $<0.001$ \\
\hline MGD grade $(0-4)$ & $0.432(0.076)$ & $<0.001$ & $0.192(0.095)$ & 0.049 \\
\hline \multicolumn{5}{|l|}{ Parameters using K5M } \\
\hline NIKBUT-first (seconds) & $-0.069(0.035)$ & 0.054 & $0.053(0.030)$ & 0.084 \\
\hline \multicolumn{5}{|l|}{ Oral parameters } \\
\hline Subjective oral score (0-3) & $0.258(0.093)$ & 0.007 & $0.074(0.067)$ & 0.279 \\
\hline NSWSF (mL/min) & $-0.122(0.047)$ & 0.011 & $-0.025(0.045)$ & 0.578 \\
\hline
\end{tabular}
time, NSWSF non-stimulated whole salivary flow, SE standard error, SSA Sjögren's-syndrome-related antigen A, SSB Sjögren's-syndrome-related antigen B

pSS and ocular involvement. Patients who had longer duration of pSS (more than 10 years) showed a higher prevalence of xerophthalmia, abnormality of Schirmer's test and/or Rose Bengal staining and other systemic involvements (parotid enlargement, lung involvement, vasculitis, or peripheral neuropathy), laboratory findings (positivity of anti-SSA/Ro antibody, anti-SSB/La antibody, and low complement $\mathrm{C} 4$ level) in a univariate analysis. But in a multivariate analysis, there were no significant correlations with xerophthalmia and abnormality of Schirmer's test and/or Rose Bengal staining that influence duration of pSS after adjusting for other factors [33]. According to this our study, patients with young age and long duration of pSS have higher risk of ocular surface damage; therefore, more careful monitor of DED is needed in those patients with pSS.

In our study, among the medical history, topical cyclosporine was associated with increased OSS in univariate analysis. This may have been due to the fact that topical cyclosporine was prescribed as treatment in order to improve the OSS in patients with severe ocular surface epithelial damage. However, after adjusting for other factors, no significant association was found in the multivariate analysis.
Of the laboratory findings in our study, positivity of anti-SSB/La antibody and RF were significant variables, and positivity of anti-SSA/Ro antibody and ANA were borderline significant variables in the univariate analysis. This result was similar with previous studies, that reported a correlation between the ocular surface parameters and serologic markers in patients with pSS $[3,15$, 16]. Serum RF, and ANA levels correlated with conjunctival staining score and total OSS in patients with pSS [15]. Furthermore, serum anti-Ro/SSA and anti-La/SSB antibodies were significantly related with clinical severity of keratoconjunctivitis sicca based on the Oxford OSS scheme in patients with pSS [16]. These studies suggested that these parameters could be considered as prognostic factors for predicting the severity and prognosis of DED in patients with pSS $[15,16]$. However, in our multivariate analysis, there were no significant laboratory findings that influenced OSS after adjusting for other factors. This was the first multivariate analysis study of association between serologic markers and OSS, and the results might differ compare to previous studies that performed a univariate analysis.

Among the oral parameters, positive subjective oral score and NSWSF were significantly related with OSS in 
the univariate analysis; however, after adjusting for other factors, no significant correlation was found. In a recent rheumatologic study, only the presence of inflammatory joint involvement among other systemic manifestations was associated with severe/very severe DED in patients with pSS (odds ratio, 2.079) [35]. Similarly, a multivariate analysis was performed in this study, thereby showing that there were no associations between other laboratory findings or oral parameters and DED severity.

Among the ocular surface parameters, FBUT and MGD were significant factors that influenced OSS in the multivariate analysis of our study. An increase in FBUT was significantly related to an increase in OSS after adjusting for other factors. The results of the Schirmer's test were significantly related with OSS in the univariate analysis, but no significant correlation was found after adjusting for other factors. According to previous study of patients with DED and ocular surface disease, FBUT is minimally invasive test and is a more reliable than the Schirmer's test. Furthermore, FBUT is strongly correlated with other ocular tests such as OSS [37]. From these results, FBUT reflected the ocular surface epithelial damage better than the Schirmer's test even in patients with pSS.

An increase in MGD grade was significantly related to an increase in OSS after adjusting for other factors. A previous study on MGD in patients with pSS reported that changes in the meibomian gland induced an increase of tear evaporation and subsequent worsening of the ocular surface desiccation [38]. Furthermore, in another study, morphological and functional features of the meibomian gland correlated with other ocular surface parameters and disease severity in ADDE, such as pSS and graft-versus-host disease [39]. According to this result, more attention is needed in pSS patients with low FBUT and severe MGD, which could cause more ocular surface epithelial damage.

There were several limitations in this study. First, the retrospective of the study might lead to unexpected various bias. Second, only a small number of patients were enrolled. Third, some of the parameters were not assessed in some of the patients. Fourth, some systemic manifestations, such as inflammatory joint involvement, were not evaluated in this study. Because of these limitations, it is necessary to conduct a prospective study in the future. However, our study is meaningful because it is the first multivariate regression analysis of factors associated with ocular surface epithelial damage in patients with pSS.

\section{Conclusions}

In conclusion, ocular surface epithelial damage in patients with pSS was associated with young age, long duration of disease, unstable tear film, and decreased meibomian gland function.

\section{Abbreviations}

SS: Sjögren's syndrome; OSS: Ocular surface staining score; pSS: Primary Sjögren's syndrome; DED: Dry eye disease; MGD: Meibomian gland dysfunction; FBUT: Fluorescein tear break-up time; NSWSF: Non-stimulated whole salivary flow; TFOS: Tear Film and Ocular Surface society; DEWS: Dry Eye Workshop; ADDE: Aqueous deficient dry eye; Th: T helper cell; IFN: Interferon; IL: Interleukin; NIKBUT: Non-Invasive Keratograph ${ }^{\oplus}$ Break-Up Time; SSA: Sjögren's-syndrome-related antigen A; SSB: Sjögren's-syndromerelated antigen B; ANA: Antinuclear antibody; RF: Rheumatoid factor; NEI: National Eye Institute; OSDI: Ocular Surface Disease Index; K5M: Keratograph ${ }^{\circledR}$ M; GVHD: Graft-versus-host disease; ACR/

EULAR: American College of Rheumatology/European League against Rheumatism classification criteria for rheumatoid arthritis; AECG: AmericanEuropean Consensus Group

\section{Acknowledgements \\ None.}

\section{Authors' contributions}

JEK and JWJ designed the study. JEK and JWJ collected and analyzed all data. NRK, HSC, KYS, TIK and JWJ reviewed the manuscript. JWJ has supervised the paper. All authors read and approved the final manuscript.

\section{Funding}

This work was supported by the Bio \& Medical Technology Development Program of the National Research Foundation (NRF) \& funded by the Korean government (MSIT) (No. 2019M3E5D1A02069616) and by INHA UNIVERSITY Research Grant

\section{Availability of data and materials}

Data sets used in this study are available from the contributing author upon reasonable request.

\section{Ethics approval and consent to participate}

The study was approved by the Institutional Review Board (IRB) of Inha University Hospital and it was complied with the tenets of the Declaration of Helsinki. No written informed consent was required as data was retrospectively collected from chart review, as approved by the reference $\mathrm{IRB}$

\section{Consent for publication}

Not applicable.

\section{Competing interests}

The authors have no financial conflicts of interest.

\section{Author details}

${ }^{1}$ Department of Ophthalmology and Inha Vision Science Laboratory, Inha University School of Medicine, 27, Inhang-Ro, Jung-gu, 22332 Incheon, South Korea. ${ }^{2}$ Department of Ophthalmology, Severance Hospital, Yonsei University College of Medicine, Seoul, South Korea.

Received: 23 August 2020 Accepted: 17 February 2021

Published online: 02 March 2021

\section{References}

1. Shimazaki J. Definition and Diagnostic Criteria of Dry Eye Disease: Historical Overview and Future Directions. Invest Ophthalmol Vis Sci. 2018;59:7-12.

2. Craig JP, Nichols KK, Akpek EK, Caffery B, Dua HS, Joo CK, et al. TFOS DEWS II Definition and Classification Report. Ocul Surf. 2017;15:276-83.

3. Liew MS, Zhang M, Kim E, Akpek EK. Prevalence and predictors of Sjogren's syndrome in a prospective cohort of patients with aqueous-deficient dry eye. Br J Ophthalmol. 2012;96:1498-503.

4. Lane HC, Callihan TR, Jaffe ES, Fauci AS. Presence of intracytoplasmic IgG in the lymphocytic infiltrates of the minor salivary glands of patients with primary Sjogren's syndrome. Clin Exp Rheumatol. 1983;1:237-9.

5. Pepose JS, Akata RF, Pflugfelder SC, Voigt W. Mononuclear cell phenotypes and immunoglobulin gene rearrangements in lacrimal gland biopsies from patients with Sjogren's syndrome. Ophthalmology. 1990;97:1599-605. 
6. Skopouli FN, Fox PC, Galanopoulou V, Atkinson JC, Jaffe ES. T cell subpopulations in the labial minor salivary gland histopathologic lesion of Sjogren's syndrome. J Rheumatol. 1991;18:210-4.

7. Katsifis GE, Rekka S, Moutsopoulos NM, Pillemer S, Wahl SM. Systemic and local interleukin-17 and linked cytokines associated with Sjogren's syndrome immunopathogenesis. Am J Pathol. 2009;175:1167-77.

8. Zoukhri D, Macari E, Kublin CL. A single injection of interleukin-1 induces reversible aqueoustear deficiency, lacrimal gland inflammation and acinar and ductal cell proliferation. Exp Eye Res. 2007;84:894-904.

9. Stern ME, Schaumburg CS, Dana R, Calonge M, Niederkorn JY, Pflufelder SC. Autoimmunity at the ocular surface: pathogenesis and regulation. Mucosal Immunol. 2010;3:425-42.

10. Ramos-Casals M, Brito-Zerón P, Seror R, Bootsma H, Bowman SJ, Dörner T, et al. Characterization of systemic disease in primary Sjögren's syndrome: EULAR-SS Task Force recommendations for articular, cutaneous, pulmonary and renal involvements. Rheumatology. 2017;54:2230-8.

11. Ozek D, Kemer OE, Omma A. Association between systemic activity Index and dry eye severity in patients with primary Sjögren syndrome. Arq Bras Oftalmol. 2019:82:45-50.

12. Mariette X, Criswell LA. Primary Sjögren's Syndrome. N Engl J Med. 2018;378; 931-9.

13. Yang S, Lee HJ, Kim DY, Shin S, Barabino S, Chung SH. The Use of Conjunctival Staining to Measure Ocular Surface Inflammation in Patients With Dry Eye. Cornea. 2019;38:698-705.

14. Wolffsohn JS, Arita R, Chalmers R, Djalilian A, Dogru M, Dumbleton K, et al. TFOS DEWS II Diagnostic Methodology report. Ocul Surf. 2017;15:539-74.

15. Lim SA, Nam S, Kwok SK, Park SH, Chung SH. Serologic Markers Are Associated With Ocular Staining Score in Primary Sjögren Syndrome. Cornea. 2015:34:1466-70.

16. Chung JK, Kim MK, Wee WR. Prognostic factors for the clinical severity of keratoconjunctivitis sicca in patients with Sjogren's syndrome. $\mathrm{Br}$ J Ophthalmol. 2012;96:240-5.

17. Vitali C, Bombardieri S, Jonsson R, Moutsopoulos H, Carsons S, Daniels T, et al. Classification criteria for Sjogren's syndrome: a revised version of the European criteria proposed by the American-European Consensus Group. Ann Rheum Dis. 2002:61:554-8.

18. Lee KW, Kim JY, Chin HS, Seo KY, Kim TI, Jung JW. Assessment of the tear meniscus by strip Meniscometry and Keratograph in patients with dry eye disease according to the presence of Meibomian gland dysfunction. Cornea. 2017:36:189-95.

19. Lemp MA. Report of the National Eye Institute/Industry workshop on Clinical trials in dry eyes. CLAO J. 1995;21:221-32.

20. Kim J, Kim JY, Seo KY, Kim TI, Chin HS, Jung JW. Location and pattern of non-invasive keratographic tear film break-up according to dry eye disease subtypes. Acta Ophthalmol. 2019;97:e1089-97.

21. Han KE, Yoon SC, Ahn JM, Nam SM, Stulting RD, Kim EK, et al. Evaluation of dry eye and meibomian gland dysfunction after cataract surgery. Am J Ophthalmol. 2014;157:1144-50.

22. Schiffman RM, Christianson MD, Jacobsen G, Hirsch JD, Reis BL. Reliability and validity of the Ocular Surface Disease Index. Arch Ophthalmol. 2000;118: 615-21.

23. Nichols KK, Foulks GN, Bron AJ, Glasgow BJ, Dogru M, Tsubota K, et al. The international workshop on meibomian gland dysfunction: executive summary. Invest Ophthalmol Vis Sci. 2011:52:1922-1929.

24. Lee H, Min K, Kim EK, Kim TI. Minocycline controls clinical outcomes and inflammatory cytokines in moderate and severe meibomian gland dysfunction. Am J Ophthalmol. 2012;154:949-57.

25. Arita R, Itoh K, Maeda S, Maeda K, Furuta A, Fukuoka S, et al. Proposed diagnostic criteria for obstructive meibomian gland dysfunction. Ophthalmology. 2009:116:2058-2063.

26. Pflugfelder SC, Tseng SC, Sanabria O, Kell H, Garcia CG, Felix C, et al. Evaluation of subjective assessments and objective diagnostic tests for diagnosing tear-film disorders known to cause ocular irritation. Cornea. 1998;17:38-56.

27. Jung JW, Park SY, Kim JS, Kim EK, Seo KY, Kim TI. Analysis of factors associated with the tear film lipid layer thickness in normal eyes and patients with dry eye syndrome. Invest Ophthalmol Vis Sci. 2016;57:4076-83.

28. Jiang $Y, Y e H, X u J$, Lu Y. Noninvasive Keratograph assessment of tear film break-up time and location in patients with age-related cataracts and dry eye syndrome. J Int Med Res. 2014;42:494-502.
29. Jung JW, Kim JY, Chin HS, Suh YJ, Kim TI, Seo KY. Assessment of Meibomian glands and tear film in post-refractive surgery patients. Clin Exp Ophthalmol. 2017:45:857-866.

30. Navazesh M. Methods of collecting saliva. Ann N Y Acad Sci. 1993;694:72-7.

31. Stonecipher KG, Torkildsen GL, Ousler GW 3rd, Morris S, Villanueva L, Hollander DA. The IMPACT study: a prospective evaluation of the effects of cyclosporine ophthalmic emulsion $0.05 \%$ on ocular surface staining and visual performance in patients with dry eye. Clin Ophthalmol. 2016;10:88795.

32. Bezzina OM, Gallagher P, Mitchell S, Bowman SJ, Griffiths B, Hindmarsh V, et al. Subjective and Objective Measures of Dryness Symptoms in Primary Sjögren's Syndrome: Capturing the Discrepancy. Arthritis Care Res (Hoboken). 2017:69:1714-23.

33. Ramos-Casals M, Solans R, Rosas J, Camps MT, Gil A, Pino-Montes JD, et al. Primary Sjögren syndrome in Spain: clinical and immunologic expression in 1010 patients. Medicine (Baltimore). 2008;87:210-9.

34. Yoon DY, Kim JH, Jeon HS, Wee WR, Hyon JY. Correlations between Tear Osmolarity and Ocular and Systemic Parameters in Primary Sjögren's Syndrome. J Korean Ophthalmol Soc. 2017:58:903-910.

35. Fernandez Castro M, Sánchez-Piedra C, Andreu JL, Taboada VM, Olivé A, Rosas J, et al. Factors associated with severe dry eye in primary Sjögren's syndrome diagnosed patients. Rheumatol Int. 2018:38:1075-82.

36. Botsios C, Furlan A, Ostuni P, Sfriso P, Andretta M, Ometto F, et al. Elderly onset of primary Sjögren's syndrome: clinical manifestations, serological features and oral/ocular diagnostic tests. Comparison with adult and young onset of the disease in a cohort of 336 Italian patients. Joint Bone Spine. 2011:78:171-174.

37. Alves M, Reinach PS, Paula JS, Cruz AAV, Bachette L, Faustino J, et al. Comparison of diagnostic tests in distinct well-defined conditions related to dry eye disease. PLoS One. 2014;9:e97921.

38. Shimazaki J, Goto E, Ono M, Shimmura S, Tsubota K. Meibomian gland dysfunction in patients with Sjögren syndrome. Ophthalmology. 1998;105: 1485-8.

39. Choi W, Ha JY, Li Y, Choi JH, Ji YS, Yoon KC. Comparison of the meibomian gland dysfunction in patients with chronic ocular graft-versus-host disease and Sjögren's syndrome. Int J Ophthalmol. 2019;12:393-400.

\section{Publisher's Note}

Springer Nature remains neutral with regard to jurisdictional claims in published maps and institutional affiliations.

\section{Ready to submit your research? Choose BMC and benefit from:}

- fast, convenient online submission

- thorough peer review by experienced researchers in your field

- rapid publication on acceptance

- support for research data, including large and complex data types

- gold Open Access which fosters wider collaboration and increased citations

- maximum visibility for your research: over $100 \mathrm{M}$ website views per year

At $\mathrm{BMC}$, research is always in progress.

Learn more biomedcentral.com/submissions 